“( 2016 IEEE. Personal use of this material is permitted. Permission from IEEE must be obtained for all other uses, in any current or future media, including

reprinting/republishing this material for advertising or promotional purposes, creating new collective works, for resale or redistribution to servers or lists, or reuse of any copyrighted component of this work in other works." 


\title{
Heart Rate Regulation During Cycle-Ergometer Exercise via Bio-Feedback
}

\author{
Ahmadreza Argha, Steven W. Su, Hung Nguyen and Branko G. Celler ${ }^{\star}$ Fellow, IEEE
}

\begin{abstract}
This paper explains our developed control system which regulates the heart rate (HR) to track a desired trajectory. The controller is indeed a non-conventional nonmodel-based proportional, integral and derivative (PID) controller. The controller commands are interpreted as biofeedback auditory commands. These commands can be heard and implemented by the exercising subject as a part of the control-loop. However, transmitting a feedback signal while the pedals are not in the appropriate position to efficiently exert force may lead to a cognitive disengagement of the user from the feedback controller. This note explains a novel form of control system regarding as "actuator-based event-driven control system", designed specifically for the purpose of this project. We conclude that the developed event-driven controller makes it possible to precisely regulate HR to a predetermined HR profile.
\end{abstract}

\section{INTRODUCTION}

A number of various strategies have been explored for the real-time control of HR during treadmill exercises such as, e.g. classical proportional, integral and derivative (PID) control [1], $\mathrm{H}_{\infty}$ control [2], [3] and model predictive control [4]. This issue has received not nearly so attention to date, for cycle-ergometer exercises. Utilizing an externally controllable automatic braked cycle-ergometer, a similar context to the treadmill exercises has been considered by adjusting the resistance of the brake rather than the pedaling rate to change the exercise level [5]. In the case of a treadmill, the treadmill speed and/or treadmill gradient are controlled directly by the controller. It means that the human does not involve actively in the control-loop (as the control system actuator) and they only need to passively respond to the variation of the controlled parameters (speed and/or gradient). This also happens for the controllable automatic braked cycle-ergometers, in which the controller controls the load on the cycling system by changing the resistance of the brake device. Computer controlled cycle-ergometers are very expensive and are not suitable for use at home as a rehabilitation device. Furthermore, our experience is that patients find increasing workload by increasing the pedalling rate more acceptable than increasing workloads at a constant pedalling rate by increasing the braking effect. Alternatively, the controller commands can be transmitted directly to the exercising subjects and, then, the human operator assumes the role of actuator of the control system.

A. Argha, S. W. Su and Hung Nguyen are with Faculty of Engineering and Information Technology, University of Technology, Sydney, PO Box 123, Broadway, NSW 2007, Australia

* B. G. Celler is currently the principal scientist in the division of computational informatics, CSIRO, Marsfield, NSW 2122, Australia Branko.cellerecsiro.au
This is commonly referred to as biofeedback. This novel framework obviates cycle-ergometers from the controllable automatic brake equipment. In this project, the air vane bike that we have selected is low cost, very sturdy and the design is particularly safe and suitable for use by frail elderly patients. In addition the cooling effect of the air vane bike can be regarded as an additional advantage.

In [6], a periodic auditory signal is utilized to motivate the exercising subject to vary their exercising rate. It means that a translating function, in accordance with the level of the controller output signal, varies the rate of auditory stimulus per minute to stimulate the subject to change their exercising rate. However, [6] ignores the position of subjects' feet in the control system design. This makes the control system a simple time-driven one, which implements the control calculations all the time at a fixed rate, which is routine in the control engineering. However, this may also lead to a cognitive disengagement of the subject form the feedback controller. Alternatively, one may consider to adjust the time duration of the auditory stimulus and/or the frequency of auditory stimulus instead. Then the rate of transmitting the auditory stimulus to the subject may be synchronized with the rate of pedalling. Implementing this idea, however, requires a new control system strategy, regarded here as actuator-based event-driven control system, which is a different counterpart of the existing sensor-based event-driven control system in the literature [7]

It should be also noticed that HR response to dynamic exercise is nonlinear and may also vary for every exercising subject, in different physical situations [6]. As a consequence, an adaptive and/or model-based control strategy might normally be exploited to deal with the problem of HR regulation. Alternatively, one may design a robust PID controller able to compensate for the interindividual differences in the dynamic HR response to work rate [5]. In this paper, an event-driven control system is proposed, in which in order to deal with the probable inaccurate controller parameters and/or individual HR profile, two anti-windup mechanisms are designed to protect the user against possible big HR fluctuations.

\section{CONTROL SYSTEM}

As explained before, in this work, the rate of output (HR) sampling is not synchronous with the rate of transmitting the controller commands (auditory signals) to the user. Here, we will discuss our new actuator-based event driven control system, in the context of these systems. 


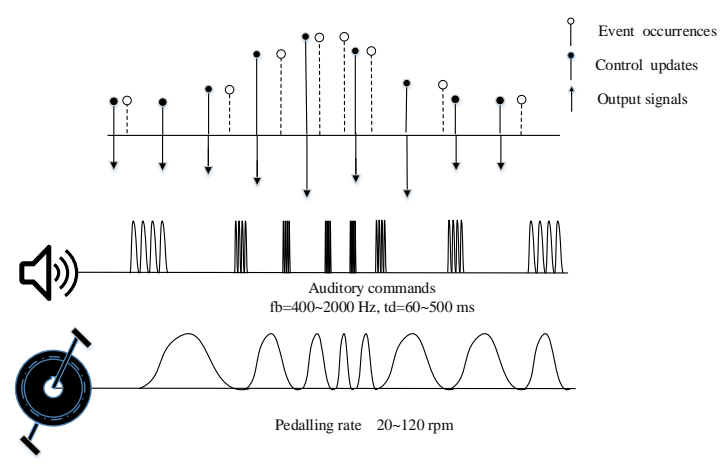

Fig. 1. Mechanism of the proposed control system.

\section{A. Actuator-based Event-driven Control Systems}

The sampling rate of the HR transmitted to the program by an ecg or pulse oximeter transducer is typically constant (1 sample per second), however, the rate of sending the commands to the exercising subject varies according to the pedaling rate.This form of control system, called here actuator-based event-driven control system, is obviously different from the sensor-based event-driven control systems; see e.g. [7]. Possibly, it could be considered somewhat as multi-rate systems, as the rates of output sampling and control input updating are different, however, since the updating rate of control input varies according to the pedaling rate of the exerciser, it could be also assumed to be an eventbased control system. Nevertheless, our proposed system is not a sensor-based event-driven one. It is because of this that the aim of this control system is to control the exercise rate through heart rate and not to control the velocity or the position of a rotary system (DC motor in the sensorbased event-driven control systems and pedaling system here) and the HR sensor signal, sampled with a constant rate independent from the pedaling rate, would not trigger the control actions. Fig. 1 gives a general view of the asynchronous signals in the designed control system.

The second method explained in [1] for the sensor-based event-driven control systems, which uses the spatial domain of the system model, could not be adapted to our work. This is, in fact, because this scheme requires the control signals and sensor (encoder) signals to be synchronous. Besides, both methods need the accurate model of system. Since, this work is aimed at developing a non-model-based PID controller, the accurate model of system is not available.

It should be noted that another kind of event-triggering mechanism with the purpose of reducing the resource utilization of its implementation has been considered [7]. This mechanism updates the control effort only when the error is bigger than a specific threshold and holds the control effort while the error is small. As an illustration, let us consider the following PID controller,

$$
u(t)=K_{p} e(t)+K_{i} \int_{0}^{t} e(\tau) d \tau+K_{d} \dot{e}(t)
$$

where

$$
e(t)=r(t)-y(t)
$$

in which $u(t), r(t), y(t), K_{p}, K_{i}$ and $K_{d}$ are control effort, reference, actual output, proportional gain, integral gain and derivative gain, respectively. Using the backward differentiation method we have,

$$
\begin{aligned}
\frac{u(k)-u(k-1)}{T_{s_{k}}}= & K_{p} \frac{e(k)-e(k-1)}{T_{s_{k}}}+K_{i} e(k) \\
& +K_{d} \frac{\frac{e(k)-e(k-1)}{T_{s_{k}}}-\frac{e(k-1)-e(k-2)}{T_{s_{k-1}}}}{T_{s_{k}}},
\end{aligned}
$$

where $T_{s_{k}}$ denotes the varying sample time. Then it follows,

$$
\begin{aligned}
u(k)= & u(k-1)+K_{p}[e(k)-e(k-1)]+K_{i} T_{S_{k}} e(k) \\
& +\frac{K_{d}}{T_{S_{k}} T_{S_{k-1}}}\left[T_{S_{k-1}}(e(k)-e(k-1))-T_{s_{k}}(e(k-1)-e(k-2))\right] .
\end{aligned}
$$

The controller in (4) is indeed suitable as a velocity form event-driven PID controller. Notice that we ignore the use of a low-pass filter to deal with high frequency measurement noise here. The event-triggering mechanism then applies based on the tracking error as

$$
\tau_{k+1}=\inf \left\{t \geq T_{s_{m}}+\tau_{k}, \text { if }|e(t)| \geq \gamma\right\},
$$

where $\tau_{k}$ is the time instant that the $k$-th controller update is generated. Also, $T_{s_{m}}>0$ and $\gamma>0$ denote the minimum sampling time of the system and threshold value, respectively. While $e(t)<\gamma$ the control effort held constant and the sample time $T_{S_{k}}$ varies. Again, note that this non-uniform updating mechanism requires that no limitation is imposed on the time instant that control efforts can apply. However, in our case as mentioned previously, the event triggering mechanism only varies the biofeedback time instants. One alternative is to neglect the triggering criteria in (5) and to use the controller in (4), which imposes additional online calculations on the processor. However, it is clear that due to the fixed sampling rate of the HR sensor, in the case that more than one update is required during each sample period, no new HR value, and hence, tracking error is available for the second and later updates. As a result, the controller can only be updated using the last available information. Consequently, it could be more likely to suffer from the windup phenomena in the system performance.

In this paper, we utilize a time-driven PID controller in the velocity form such as

$$
u(k)=u(k-1)+\Delta u(k),
$$

where

$$
\begin{aligned}
\Delta u(k)= & K_{p}[e(k)-e(k-1)]+K_{i} T_{s} e(k) \\
& +\frac{K_{d}}{T_{s}}[e(k)-2 e(k-1)+e(k-2)],
\end{aligned}
$$

in which $T_{s}$ is a fixed sampling rate and is equivalent to the sampling period of the HR sensor. Then, whenever an event occurs (the left or right pedal pass the reed sensor), the last prepared update will be transmitted as a biofeedback signal. 


\section{B. HR profile}

The HR profile, in this work, is selected in three stages. In the first stage, called the warm up period, we aim to gradually increase the user HR from their normal HR $\left(H R_{n}\right)$ to the exercise $\mathrm{HR}\left(H R_{e}\right)$ which then remains constant for a while $\left(T_{e}\right)$. The warm up slope is

$$
\theta_{w}=\frac{H R_{e}-H R_{n}}{T_{w}},
$$

in which $\theta_{w}$ represents warm up slope and $T_{w}$ shows the warm up time duration. The warm up time duration $T_{w}$ is indeed used to avoid an excessively rapid increase in the HR. The second stage called the exercise period is indicated by $T_{e}$, which may be computed according to the maximum $\mathrm{HR}$. The maximum HR $\left(H R_{\max }\right)$ commonly is characterized by the well-known Haskell and Fox formula as:

$$
H R_{\max }=220-\text { age. }
$$

We set then the exercising $H R_{e}$ as $0.65 \sim 0.85$ of $H R_{\max }$, depending on the subjects level of fitness and/or risk profile. The application of the third cooling down or recovery stage $\left(T_{c}\right)$ is basically to prevent possible venous pooling and reduced venous return to the heart from an excessively abrupt termination in exercise.

\section{Auditory convertor}

As mentioned, in order of facilitating the user applying more or less effort at the right time, it is found that the combination of varying both the time duration and the frequency is an effective method.

In this method a longer time duration and lower frequency is used to motivate the subject to do the pedalling slowly and vice versa at the quick pedaling. Moreover, the range of time duration and frequency which can be used are limited. We know that the time durations more than a specific value result in the so-called aliasing phenomena. Additionally, the time durations which are less than a specific value may not be heard by the subjects. This issue also holds for the auditory signals' frequency. As a result, we selected the following range of time duration and frequency for the auditory stimulus as $60 \sim 500 \mathrm{~ms}$ and $400 \sim 2000 \mathrm{~Hz}$, respectively. Therefore, the auditory converter contains two saturation functions,

$$
t_{d}=\operatorname{sat}\{g(u(k))\}=\left\{\begin{array}{lr}
\lambda_{1} & g(u(k)) \leq \lambda_{1} \\
g(u(k)) & \lambda_{1}<g(u(k))<\lambda_{2} \\
\lambda_{2} & g(u(k)) \geq \lambda_{2}
\end{array}\right.
$$

where $t_{d}$ is the time duration of the auditory signals and $g(u(k))$ is a scaling function which converts the range of $u(k)$ to the range of time duration, $\lambda_{1}=60 \mathrm{~ms}, \lambda_{2}=500 \mathrm{~ms}$, and

\section{(11)}

$$
f_{b}=\operatorname{sat}\{h(u(k))\}=\left\{\begin{array}{lr}
\gamma_{1} & h(u(k)) \leq \gamma_{1} \\
h(u(k)) & \gamma_{1}<h(u(k))<\gamma_{2} \\
\gamma_{2} & h(u(k)) \geq \gamma_{2},
\end{array}\right.
$$

where $f_{b}$ is the frequency of the auditory signals and $h(u(k))$ is also a scaling function which converts the range of $u(k)$ to the range of appropriate frequencies of the auditory signal, $\gamma_{1}=400 \mathrm{~Hz}$ and $\gamma_{2}=2000 \mathrm{~Hz}$.

\section{Implementing anti-windup mechanisms}

The so-called windup phenomena may happen due to the utilizing the integral action in the controller and saturation in the system actuators. Here, as the exercising subject plays the role of system actuator, and has its exercise limitations, windup could occur. Indeed, during the windup phenomena the closed-loop control system cannot implement its correct control strategy and the actuators will perform at their limits independently of the system outputs measured. In the case of utilizing an integrator in the controller, the error will be integrated continuously for a long period before things return to normal. Hence, control effort may increase to a very large value. Thus the PID controller may cause large transients while the system actuator saturates.

In our case, if the exercising subject inadvertently or deliberately chooses not to follow the controller commands, a big tracking error will be generated, and the controller will require a huge effort to compensate for the absent effort. This may result in a big fluctuation in the HR of the subjects. If HR profile (such as warm up slope $w_{s}$ in (8) and target HR $\left.\left(H R_{e}\right)\right)$ is not determined accurately for each subject, considering the maximum work-rate that the subject can do whilst pedaling continuously, a collapse in the closed-loop mechanism could occur. Even if the target HR and warm up slope are set appropriately according to each subject's condition, the subject may deliberately choose to stop the exercise for a short while.

In the conventional control technology, to deal with this problem, a number of anti-windup schemes have been developed, which need to know and use the actuator limitations. Here, however, the exercising subjects activity limitations are not easily obtained. Also, it is not always possible to determine that the subject's unresponsiveness is inadvertent or deliberate. In order to address the problem, the HR profile needs to be more dynamic. In other words, in the cases that big tracking errors are noted (bigger than a specific threshold), the HR profile could be temporarily held constant to prevent big efforts being required of the subjects. We thus revise the HR profile as follows,

$$
w_{s}= \begin{cases}\frac{H R_{n}-H R_{e}}{T_{w}} & \text { if } e(k) \leq \delta, \\ 0 & \text { if } e(k)>\delta,\end{cases}
$$

where $\delta>0$ denotes a certain threshold. Notice that the warm up duration will then increase to $\bar{T}_{w}=T_{w}+T_{a}$ which $T_{a}$ is the summation of all time periods that $e(t)>\delta$ and $w_{s}=0$. This could avoid any big fluctuation in the HR of the subject.

On the other hand, since the designed control system contains an auditory converter (biofeedback generator) it is also possible that both converters (audio frequency and time duration) reach their limitations, even with accurate definition of the translating functions $t_{d}$ and $f_{b}$ in (10) and (11), respectively. This could also result in windup phenomena. Note that the velocity form PID control strategy 
first computes $\Delta u(k)$, see (6), which is then integrated via an integrator. In some industrial cases this integrator is a part of the actuator and as a result while the actuator is in the saturation mode the windup would be avoided automatically. But, when the integrator is designed in the controller, the windup would be addressed by assuming $\Delta u(k)=0$ during the saturation. Thus, we define

$$
\begin{cases}u(k)=u(k-1)+\Delta u(k), & \text { if }\left\{\begin{array}{l}
\lambda_{1}<g(u(k))<\lambda_{2} \& \\
\gamma_{1}<h(u(k))<\gamma_{2}
\end{array}\right. \\
u(k)=u(k-1), & \text { otherwise. }\end{cases}
$$

\section{Methodology And EQuipments}

In this project, we utilized a Nonin 4100 Pulse Oximeter to collect the HR data during the exercise. Besides, the cycleergometer that we used in this work is an air vane exercise bike, which is especially well adapted for safe exercise by the frail elderly. Since it has a big supporting structure, which can be used by the exercising patient to stabilize themselves during exercising, the finger pulse oximeter is almost completely stable during exercise and generates minimal artefact. We have developed an acquisition system using the National Instrument LabVIEW which provides easy synchronization and graphical user interface.

In addition, we attached two reed switches and magnets to the crank shaft of pedals of exercise bike, in order to provide a pulse whenever a full revolution has been completed. In the Labview program, a time-delay parameter may be set to help the user to adjust the time-delay between the position of the pedal sensed by the sensor and the time when the command will be sent. This parameter in the designed software is useful since we can set the point where the user can most effectively apply force to the pedals.

\section{EXPERIMENTAL RESUlTS}

This section will describe the results and performance of the controller designed for this project. A wide range of experiments were carried out on six healthy male subjects during cycling exercise. The HR profile was defined in 3 stages; warm up, exercise and cool down, where each lasted 5,5 and 7 minutes for each subject, respectively. Moreover, the reference value of $125 \mathrm{bpm}$ is our desired aerobic training HR for all subjects during the exercise stage.

We also implemented a large number of experiments primarily in order to find the average transfer function of HR response so that eventually a PID control tuning has been obtained which is more robust against the inter-individual differences in the dynamic HR response to work rate. The PID controller parameters have been accordingly tuned to $K_{p}=3, K_{i}=0.03$ and $K_{d}=0.02$. The tracking performance of this controller for one subjects is demonstrated in Figs. 2. It can be concluded from the result that the designed PID controller can efficiently drive the subject to track the desired HR profile.

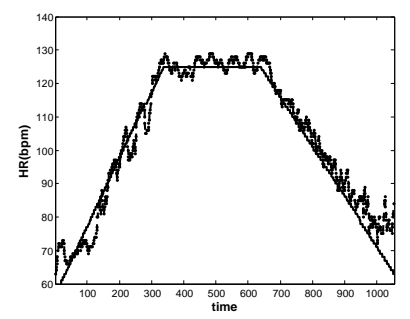

Fig. 2. HR profile tracking during cycling using PID controller

\section{Conclusions}

In this study, a new control system called actuator-based event driven control system has been designed to help the exercising subjects to track a preset HR profile. The advantage of this study, compared to other published data, is that in our automated system the biofeedback signals are synchronized with respect to the positions of the pedals. This work shows that adjusting the time duration and the frequency of the auditory signals, rather than the rate of the auditory signals per minute is more effective to force the subjects HR to track the profile. However, this required to design a new control scheme instead of the conventional time-driven control systems used in the literature. Besides, this control system has been equipped with an anti-windup mechanism to avoid the possible wind-up phenomena which may occur in the system.

\section{REFERENCES}

[1] T. Kawada, G. Sunagawa, H. Takaki, T. Shishido, H. Miyano, Miyashita, T. Sato, M. Sugimachi and K. Sunagawa, "Development of a servo-controller of heart rate using a treadmill," Japanese Circulation Journal, vol. 63, pp. 945-950, 1999.

[2] T. M. Cheng, A. V. Savkin, B.G. Celler, S. W. Su, and L. Wang, "Nonlinear modeling and control of human heart rate response during exercise with various work load intensities," IEEE Transactions on Biomedical Engineering, vol. 55, no. 11, pp. 2499-2508, 2008.

[3] S. W. Su, L. Wang, B. G. Celler, A. V. Savkin, and Y. Guo, "Identification and control for heart rate regulation during treadmill exercise," IEEE Transactions on Biomedical Engineering, vol. 54, no. 7, pp. 1238-1246, 2007.

[4] S. W. Su, S. Huang, L. Wang, B. G. Celler, A. V. Savkin, Y. Guo, and T. Cheng, "Nonparametric hammerstein model based model predictive control for heart rate regulation," in Proc. IEEE Eng. Med. Biol. Soc., pp. 2984-2987, 2007.

[5] T. Kawada et al, "Development of a servo-controller of heart rate using a cycle ergometer," Heart Vessels, vol. 14, pp. 177-184, 1999.

[6] D. Baig, F. Javed, A. V. Savkin and B. G. Celler, "An Adaptive $\mathrm{H}_{\infty}$ Control Design for Exercise-Independent Human Heart Rate Regulation System," in Proc. the 9th IEEE International Conference on Control and Automation (ICCA), Santiago, Chile, Dec. 19-21, 2011.

[7] W. P. M. H. Heemels, J. H. Sandee, and P. P. J. Van Den Bosch, "Analysis of event-driven controllers for linear systems," International Journal of Control, vol. 81, no. 4, pp. 571-590, 2008. 